\title{
Morphology of crystalline Nylon-610 membranes prepared by the immersion-precipitation process: competition between crystallization and liquid-liquid phase separation
}

\author{
Tai-Horng Young ${ }^{\mathrm{a}}$, Dar-Jong Lin ${ }^{\mathrm{b}}$, Jy-Jye Gau ${ }^{\mathrm{b}}$, Wen-Yuan Chuang ${ }^{\mathrm{a}}$, Liao-Ping Cheng ${ }^{\mathrm{b}, *}$ \\ ${ }^{a}$ Center for Biomedical Engineering, College of Medicine, National Taiwan University, Taipei 10016, Taiwan \\ ${ }^{\mathrm{b}}$ Department of Chemical Engineering, Tamkang University, Tamsui, Taipei Hsien 25137, Taiwan
}

Received 1 July 1998; received in revised form 18 September 1998; accepted 14 October 1998

\begin{abstract}
Nylon-610 membranes were prepared at $25^{\circ} \mathrm{C}$ by direct immersion of various dope solutions into either formic acid/water or 1-octanol bath. Depending on the dope and bath conditions, the precipitated membranes demonstrated characteristics derived from crystallization and/ or liquid-liquid phase separation during the precipitation process. As a good dope solution was immersed in a harsh bath, e.g., water, precipitation occurred initiated by liquid-liquid phase separation. The formed membrane exhibited a cellular structure similar to that commonly observed in amorphous membranes. Alternatively, when a metastable dope (with respect to crystallization) was immersed in a soft bath containing a substantial amount of formic acid, crystallization dictated the precipitation process to yield bi-continuous, particulate membranes. Membranes with extensive macrovoids were observed, in the event that the dope contained a large amount of solvent. In the latter case, precipitation took place immediately after immersion, consistent with Strathmann and Smolder's results for several membrane forming systems. In addition, skinless microporous membranes were prepared by precipitation of the dope solutions in a 1-octanol bath, in which precipitation occurred slowly and the formed membrane was composed of "sheaf-like" crystallites that interlocked into a homogeneous bi-continuous network (C) 1999 Elsevier Science Ltd. All rights reserved.
\end{abstract}

Keywords: Membrane; Nylon; Crystallization

\section{Introduction}

Aliphatic polyamides, such as Nylon-6, -66 and -46 are crystalline polymers that have long been used to synthesize porous membranes by the isothermal immersion-precipitation method [1,2,3]. Marinaccio [1] and Paul [2] have shown that skinless microporous Nylon membranes could be synthesized by precipitation of Nylons from formic acid aqueous solutions. These membranes are nowaday employed extensively in various separation processes. Kesting addressed the Nylon-6 and -66 membranes as the "state of the art" microfiltration membranes [4]. Fang investigated the potonation of Nylon-6 in hydrochloric acid solutions and found that the molecular weight of polymer in these dope solutions depended upon the dope's age; the latter in turn affected the morphology of the fomed membrane [5]. Huang [6] used Nylon-6 and Lai [7] used Nylon-4 asymmetric membranes,

\footnotetext{
* Corresponding author.

E-mail address: lpcheng@ @igma.che.tku.edu.tw (L.-P. Cheng)
}

respectively, to enrich alcohol from aqueous solutions in the pervaporation process.

Although there have been extensive studies on the formation mechanism of amorphous membranes, understanding of crystalline polyamide membranes is customarily based upon empiricism inferred from correlation between preparation conditions and the properties of the resultant membranes. In fact, formation of microporous polyamide membranes has often been considered as a matter of "art". Recently, Cheng [8,9,10] and Bulte [11] began to stress the role of crystallization in the precipitation process and put forward the concepts of incipient dopes and soft baths that formed crystalline morphology. In a series of publications $[9,10,12]$, Cheng proposed that formation of crystalline membranes might be treated as a competition between the liquid-liquid phase separation and crystallization processes and that the sequence of these events determined the resultant membrane structures. When crystallization initiated and dominated the phase separation process, microporous membranes were formed, which were composed of crystalline particles in the shape of stick, sheaf, or sphere, 
depicting different stages of spherulitic growth [13]. These crystallites interlocked into a uniform bi-continuous network. On the other hand, when liquid-liquid phase separation preceded crystallization, asymmetric membranes were produced whose structure was similar to that of an amorphous asymmetric membrane, featuring a thin skin and a cellular porous bulk. In the intermediate cases, the membranes exhibited mixed morphologies characterized both by crystalline particles and cellular pores.

In the present work, formation of Nylon-610 membranes by isothermal immersion-precipitation was investigated. The morphology of these membranes, in general, depended on the dope and bath conditions in the same trends as the Nylon-66 and -6 membranes [9,10]. However, there were two major differences: (1) Nylon-610 did not precipitate into skinless uniform structure in water-formic acid baths. Such was the case even for precipitating an incipient dope (metastable with respect to crystallization) in an extremely soft bath (e.g., 60 wt.\% formic acid-water); (2) Significant finger-like macrovoids were formed when the dope contained a large amount of solvent. In this case, precipitation took place immediately after immersion consistent with Strathmann's [14] and Smolder's [15] results for several membrane formation systems. These macrovoids had porous walls that were composed of "sticklike" crystallites. Such a structure has never been reported in the literature. Finally, a skinless microporous membrane was made by precipitating the dope solutions in a 1-octanol bath. This membrane was composed of "sheaf-like" crystallites that interlocked into a homogeneous bi-continuous network, suggesting that crystallization rather than liquid-liquid phase had dominated the phase separation process.

\section{Experimental}

\subsection{Materials}

All materials were obtained from commercial sources and used as received. Poly(hexamethylene sebacamide) (Nylon610 , Ultramide BASF, intrinsic viscosity $=1.147 \mathrm{gm} / \mathrm{dl}$, $\mathrm{M} \eta=33,000 \mathrm{gm} / \mathrm{mole}$ ) was received in pellet form. Formic acid (FERAK, 98\% by weight, $r=1.22 \mathrm{~g} / \mathrm{ml}$ ) was used as the solvent. Both water (distilled, deionized) and 1-octanol (Reidel-de Haen, reagent grade, $d=0.82 \mathrm{~g} /$ $\mathrm{ml})$ were chosen as the nonsolvent for Nylon-610.

\subsection{Membrane formation}

A dope solution was uniformly cast on a glass surface (ca. $250 \mu \mathrm{m})$. It was then immersed at $25^{\circ} \mathrm{C}$ into a coagulation bath composed of specific amounts of non-solvent (water or 1-octanol) and solvent (formic acid). After precipitation has completed (ca. $20 \mathrm{~min}$.), the membrane was removed from the bath. The nonsolvent and the residual solvent in the membrane were removed by a sequence of washing steps.
Finally, the formed membrane was held tightly in a press between two sheets of filter papers and then dried at $40^{\circ} \mathrm{C}$.

\subsection{Membrane characterization}

Several methods were employed to characterize the membranes, as shown below.

1. Structures of the membrane in the top, bottom, and edge views were examined using a scanning electron microscope (HITACHI, S-800). The cross-section of a membrane was obtained by fracturing the membrane in liquid nitrogen and then coated with gold-palladium.

2. The melting endotherms of both the polymer pellets and the formed membranes were determined by differential scanning calorimeter (DSC 200, Netzch, Germany) at the rate of $10^{\circ} \mathrm{C} / \mathrm{min}$ over the range of $40-250^{\circ} \mathrm{C}$.

3. Wide angle X-ray diffraction (Rigaku D/MAX-2000) experiments of the membrane were carried out to obtain the crystal structure of the membranes.

4. Water wettabilities of various membranes were measured following Pall's method [2]. A drop of distilled water $(2 \mu \mathrm{l})$ was deposited on the surface of a membrane placed on the stage of an optical comparator. The time was recorded for the drop to be completely absorbed into the membrane.

\subsection{Measurement of precipitation time}

Light transmission experiments were performed to measure the time of the onset of phase separation in the immersion process. A collimated light beam was directed to the membrane solution immersed in a nonsolvent bath. The light transmittance is recorded with a data acquisition system. The initial precipitation time is identified as the time at which transmission intensity starts to decrease. The detailed experimental setup and procedures are shown in a previous publication [10].

\section{Results and discussion}

\subsection{Dope, bath, and phase equilibrium isotherms}

The phase diagram at $25^{\circ} \mathrm{C}$ of the ternary system, water/ formic acid/Nylon-610, is shown in Fig. 1 [8]. As Nylon610 is a crystalline polymer, phase separation may occur in the forms of crystallization and/or liquid-liquid phase separation. The crystallization line represents the composition of the liquid phase, which coexists in equilibrium with the Nylon-610 crystallites. A solution below this line will eventually undergo a crystallization induced solid-liquid demixing. The region of liquid-liquid phase separation is defined by the binodal phase envelope. A solution within this region will separate into two liquid phases in equilibrium. The compositions of the two phases are jointed by 


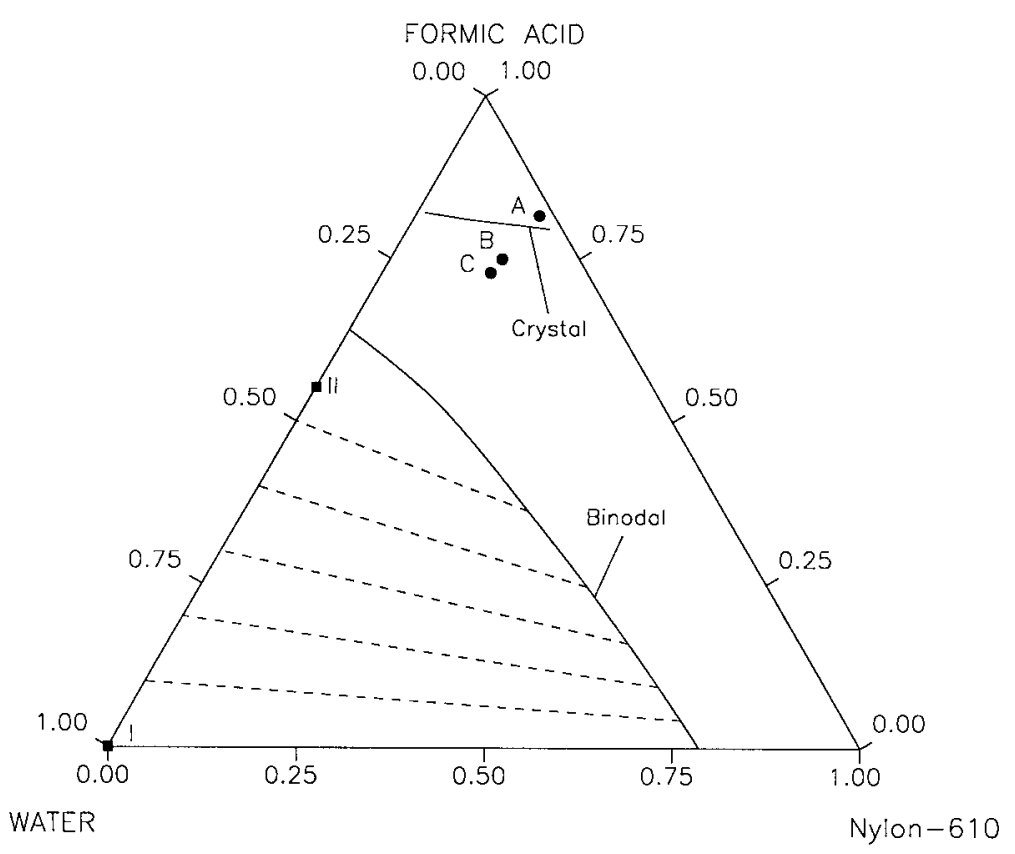

Fig. 1. Ternary phase diagram of water-formic acid-Nylon-610 system at $25^{\circ} \mathrm{C}$.

the tie lines. As the binodal is below the crystallization line, both types of phase separations can occur for solutions within the binodal region. It has been shown previously that the competition between these two types of phase separations during the immersion-precipitation process will determine the structure of the formed membranes $[9,10,12]$.

In this report, three representative dopes and two baths will be discussed. Their compositions are shown in Fig. 1. Dope " $\mathrm{A}$ " is a homogeneous solution which contains only a small amount of non-solvent (water $=1.7 \mathrm{wt} . \%$, formic acid $=83.3 \mathrm{wt} . \%$, Nylon-610 $=15 \mathrm{wt} . \%$ ). The formic acid in this solution acts as a proton donor which solvates Nylon-610 through hydrogen bonding. Dope "B" (water = $8.75 \mathrm{wt} . \%$, formic acid $=77.56 \mathrm{wt} . \%$, Nylon-610 $=$ $13.69 \mathrm{wt} . \%$ ) and "C" (water $=11.0 \mathrm{wt} . \%$, formic acid $=75.7$ wt. $\%$, Nylon- $610=13.3$ wt. $\%$ ) are supersaturated with respect to crystallization; i.e., they are below the crystallization line. As dopes "B" and "C" would gel upon standing at room temperature, they have to be heated before immersing at $25^{\circ} \mathrm{C}$ into the bath. These supersaturated dopes have often been described as a mixture that contains a large amount of pre-nucleation aggregates. As these dopes are on the edge of precipitation, they are termed incipient dopes by Pall [2]. Bath "I" (i.e., pure water) in Fig. 1, is a typical harsh nonsolvent for most polymers. In this bath, phase separation takes place rapidly after the immersion procedure. In contrast, bath "II" (60 wt.\% formic acid) is a very "soft" precipitant with respect to Nylon-610. As a dope is immersed in this bath, the exchange of solvent and nonsolvent proceeds slowly, leading to a delayed phase separation situation [8-10] (exceptions can occur when highly supersaturated dopes are used).

\subsection{Nylon-610 membrane with skinned sponge-like structure}

Fig. 2 presents the edge (a), top (b), and bottom (c) views of the membrane prepared by immersing dope " $\mathrm{A}$ " in bath "I". The cross section consists of irregular voids enclosed in a polymer mat. These voids are largely of identical size (ca. $1-2 \mu \mathrm{m}$ dia.) and are distributed uniformly in the membrane cross section. Such a porous morphology is similar to the so-called "sponge structure" typically observed in amorphous membranes (e.g., polysulfone [16], polycarbonate [17], and cellulose acetate [18] membranes) that are produced by the liquid-liquid demixing process $[12,16,18,21,22]$. This implies that liquid-liquid phase separation has taken place earlier than crystallization and eventually dominated the membrane structure, even though the crystallization line was crossed right after the immersion procedure. It appeared that the long induction time associated with polymer crystallization (nucleation and growth) rendered the latter process to occur only after completion of liquid-liquid phase separation. In the liquid-liquid demixing process, nucleation of liquid micells composed of formic acid and water commenced when the membrane solution entered the binodal phase envelop. These micells were in equilibrium at their interfaces with the surrounding polymer-rich gel phase. Radial growth of the micells was made possible by influx of solvent and nonsolvent in the gel phase. This growth continued until the polymer-rich phase fused and solidified. Occasionally, when the gel layer between micells was thin and mechanically weak, the cell wall might break and the cells became interconnected. This is often referred to as the phase inversion process in the literature [22]. As the polymer-rich gel was always in a state 

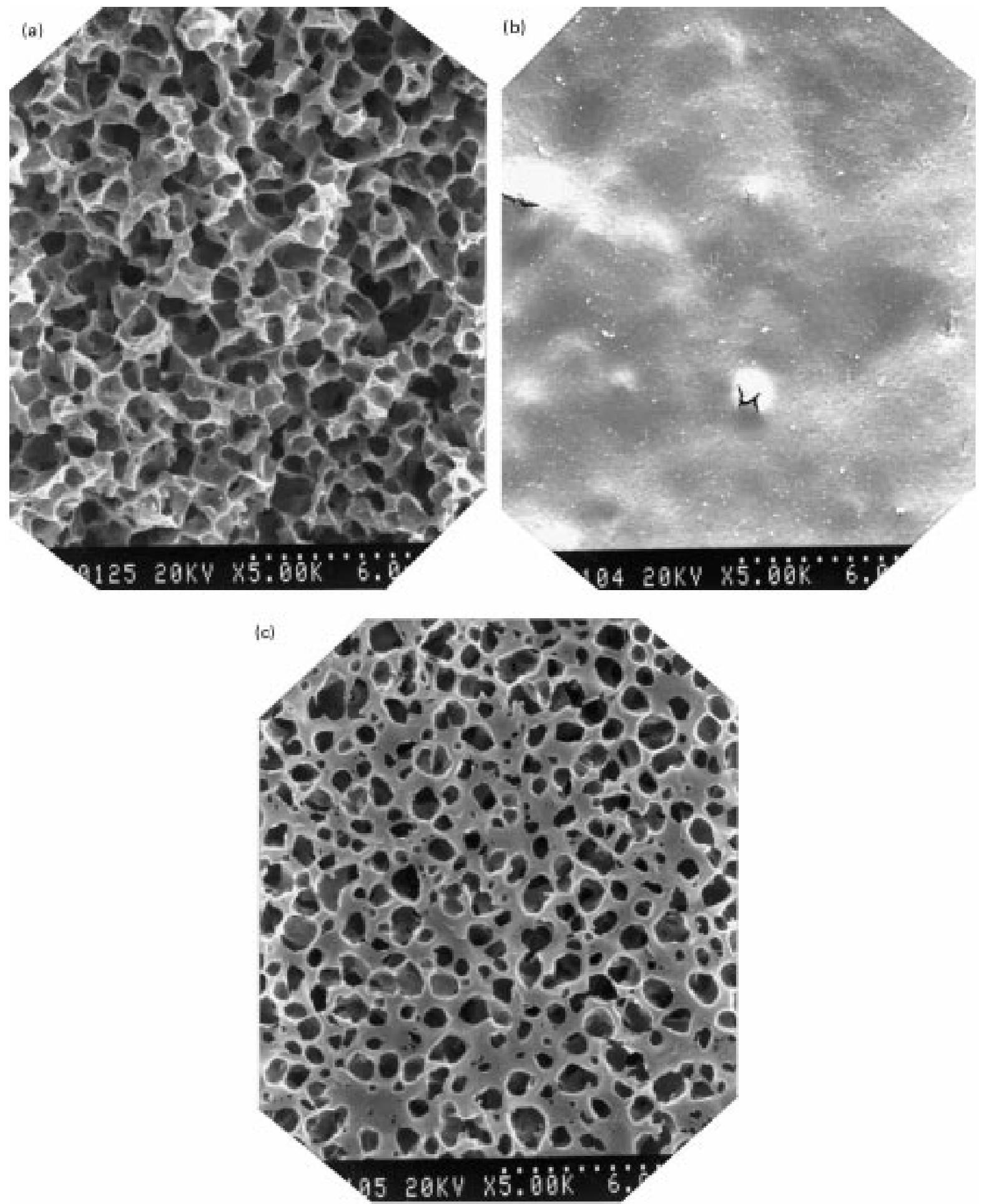

Fig. 2. SEM photomicrographs of the membrane prepared by immersing dope A in bath I. (a) edge view; (b) top view; (c) bottom view.

supersaturated with respect to crystallization (see the phase diagram in Fig. 1), it eventually crystallized into the solid matrix. Such is evident from the roughened and dendritic pore surfaces shown in Fig. 2(a). Also, because solvent and nonsolvent were rejected into the liquid micells during polymer crystallization near the pore wall, the pore was distorted from a perfect sphere. The DSC thermogram of the membrane is shown in curve " $\mathrm{B}$ " of Fig. 3. The heat of fusion of the membrane is virtually equal to that of the melt-crystallized Nylon-610 pellets (curve A) and the membrane has a slightly higher melting temperature than the polymer pellets. The X-ray diffraction patterns of this 


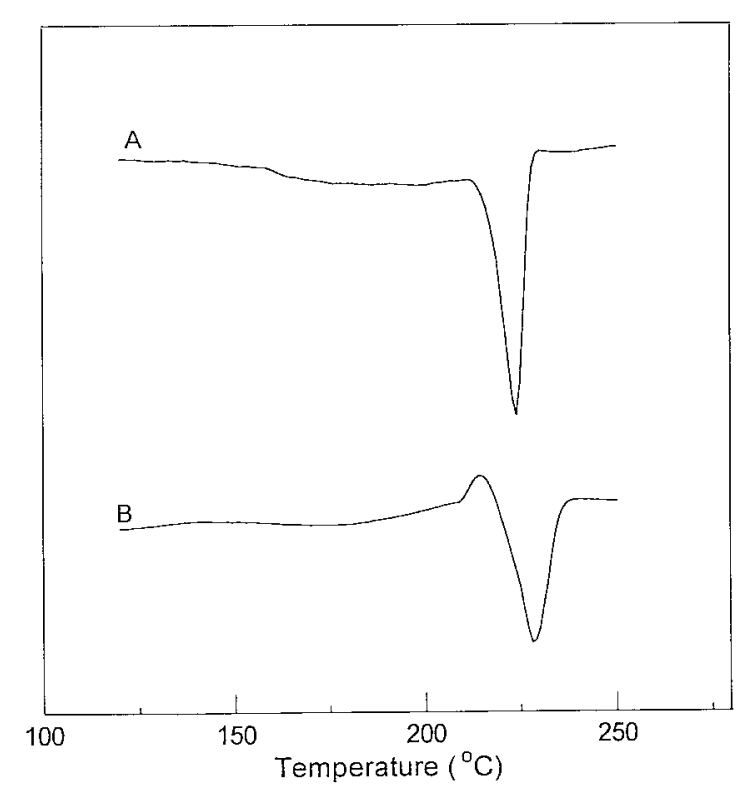

Fig. 3. DSC thermograms of the Nylon-610 pellets and the membrane prepared by immersing dope $\mathrm{A}$ in bath $\mathrm{I}$. curve $\mathrm{A}: T_{\mathrm{m}}$ (peak value) $=$ $224^{\circ} \mathrm{C}, H=47.58 \mathrm{~J} / \mathrm{g}$; B: $T_{\mathrm{m}}$ (peak, value) $=228^{\circ} \mathrm{C}, H=45.94 \mathrm{~J} / \mathrm{g}$.

membrane is shown in Fig. 4 together with those of the other membranes prepared in different dope or bath conditions. Comparing with the crystallographic data given in the literature $[13,19,20]$, the crystallites in these membranes appear to be of ' $\alpha$ ' structure that are oriented in a triclinic unit cell. As the membrane exhibited a cellular structure, these results suggests that crystallization occurred at the late stage of immersion-precipitation, wherein liquid-liquid phase separation has completed and the cellular structure has largely been fixed.

The top surface of this membrane, as shown in Fig. 2(b), forms a continuous dense skin. There are no appreciable pores except for several irregular crevices that might be caused by impingement of high energy electron beam $(20 \mathrm{kV})$ during SEM observation. Fig. 2(b) indicates that liquid-liquid phase separation did not occur in the membrane top surface. This is consistent with the fact that interfacial polymer concentration increased markedly soon after contact with the harsh bath [10,15,23]. Polymer formed a stiff gel in this region, which precluded any possibility of nucleation of liquid micells. Besides, the top surface was ordinarily considered to be in equilibrium with the precipitation bath; hence, there was no tendency for liquid-liquid demixing to take effect $[10,15,23]$ and a tight skin layer was formed on the top surface region.

The structure of the bottom surface is presented in Fig. 2(c). It is also composed of separate cellular pores that are embedded in a continuous polymer phase. These pores, unlike those in the membrane cross section, have the shape of truncated globes (ca. $1 \mu \mathrm{m}$ diameter), suggesting that liquid micells might have been nucleated directly on the glass surface during the precipitation process. The nascent micells, then, grew radially into the membrane solution to form the truncated cellular structure. The pore walls also demonstrated a dendritic feature as in the membrane interior. On the bottom surface, polymer crystallites grew against the glass surface leading to the flattened morphology as shown in the figure.

\subsection{The effect of formic acid content in the bath}

When immersion-precipitation is carried out in a soft bath containing a high concentration of solvent, the rate of solvent-nonsolvent exchange across the bath-membrane interface drops off and precipitation is delayed. A number of studies have indicated that in this case the membrane solution will enter the binodal slowly $[10,15,23]$. Therefore, crystallization may occur together with liquid-liquid phase separation to yield membranes showing characteristics from both crystallization (i.e., particulate crystallites) and liquidliquid phase separation (i.e., cellular pores). Such a case is manifested in Fig. 5. The membrane was prepared by immersing dope "A" in a $60 \mathrm{wt} . \%$ formic acid/water bath, i.e., point "II" in Fig. 1. The top surface of this membrane is shown in Fig. 5(a). Unlike that observed in Fig. 2(b), this surface is not very tight and it exhibits a polygonal structure similar to the planar spherulite commonly observed in melt crystallized polyamide films $[9,11,13]$. This implies that, during precipitation, crystalline nuclei were formed just underneath the top surface wherein liquid-liquid phase separation could not occur. These nuclei then grew two dimensionally following the habits of spherulites until their fronts impinge each other to form the polygonal structure with linear grain boundaries. The central region of each crystal grain, i.e., the nucleation site, bulged up as a result of rejection of impurities (basically, solvent and nonsolvent) into the bath, which made the crystallite looked like the "Vietnamese hat".

Fig. 5(b) shows the cross-sectional structure of the membrane. The cross section exhibits a uniform porous structure. The cellular pores (ca. $1 \mu \mathrm{m}$ dia.) here are not that distinctive as those shown in Fig. 2(a), and they are also distorted from being spherical. Crystallites (ca. $1 \mu \mathrm{m}$, in length) are observed on the pore walls and all of the pores are interconnected to form a bi-continuous structure. Such a mixed morphology of crystallization and liquid-liquid phase separation suggests that these two types of phase transformations have begun at a close time frame. In other words, soon after liquid micells were formed, crystallization commenced on the cell boundary. During growth of polymer crystallites, solvent and nonsolvent were rejected into the micells, leading to the distorted and irregular cellular pores. The bottom surface of this membrane, as presented in Fig. 5(c) also shows strong evidences of both types of phase separations. In contrast to the separate cellular pores shown in Fig. 2(c), the pores here are inter-connected and the cell boundaries are very thin, some of which even develop into sharp stick-like crystallites. 


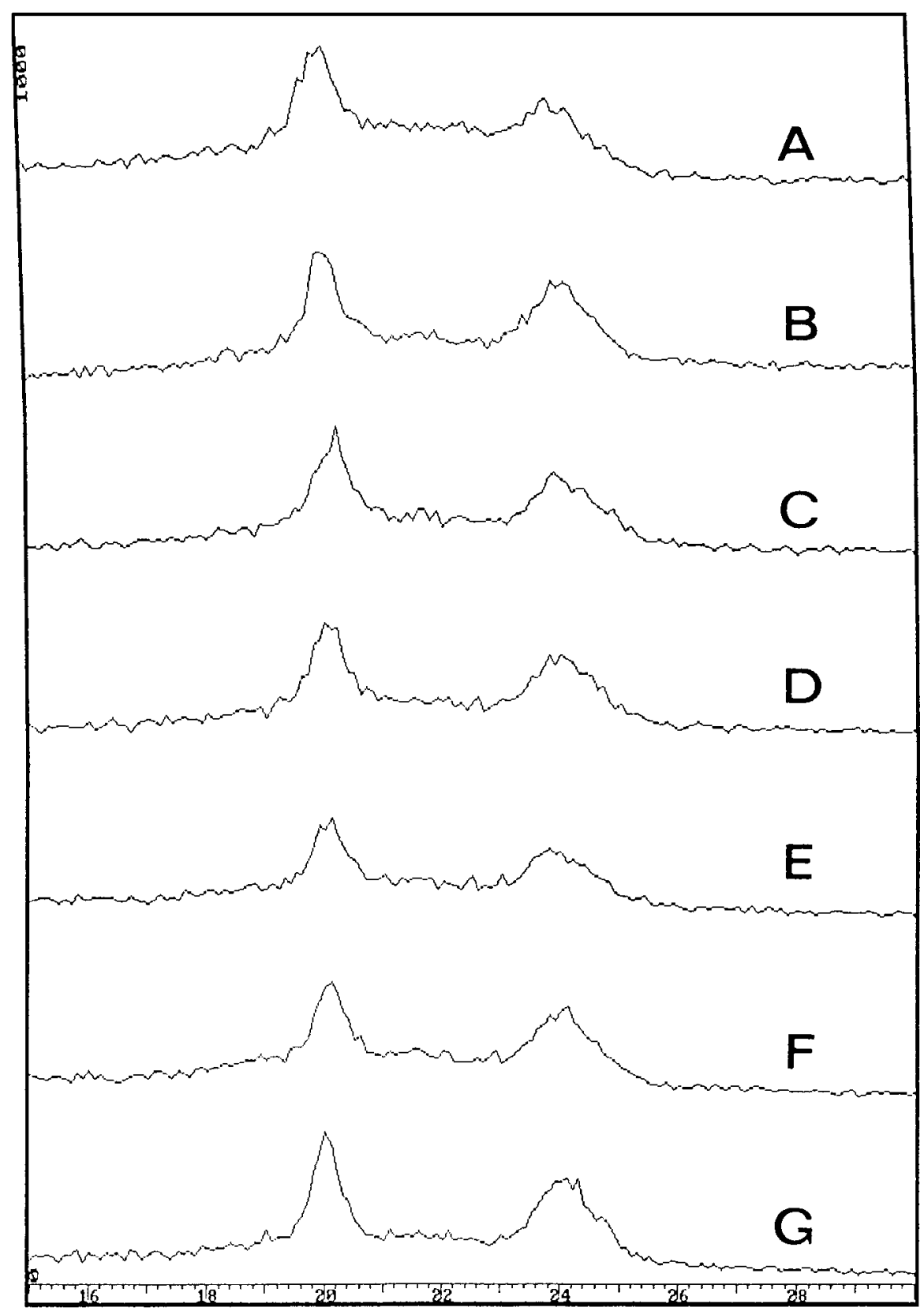

Fig. 4. X-ray diffraction scans of various membranes. A: dope 'A' in bath 'I'; B: dope 'A' in bath 'II'; C: dope 'B' in bath 'I'; D: dope 'B' in bath 'II'; E: dope 'C' in bath 'I'; F: dope 'C' in bath 'II'; G: dope 'A' in 1-octanol.

\subsection{The effect of water content in the dope}

As the incipient dope "B" was immersed in bath "I", the structure of the formed membrane exhibited similar features to that prepared by immersing dope " $\mathrm{A}$ " in the same bath (cf., Fig. 2). In this event, the membrane structure was only slightly affected by the water content in the dope. However, as dope "B" was immersed in bath "II", the ordinarily sluggish crystallization process (compared to liquid-liquid phase separation) now began to dominate the precipitation process and a largely uniform microporous membrane was obtained. The SEM photomicrographs of this membrane are illustrated in Fig. 6. The cross section of this membrane, as shown in Fig. 6(a), is composed of stick or sheaf-like crystalline particles (ca. $1.5 \mu \mathrm{m}$, length), which interlock to form a totally open bi-continuous network. Thread-like textures $(0.05 \mu \mathrm{m}$, estimated thickness $)$ can also be observed on the crystallites. Structures characteristic of liquid-liquid phase separation (i.e., cellular pores) are not evident in this cross-sectional region; all voids appear to be random and have ill-defined shapes. Compared with the membrane prepared from dope A in the same bath (cf., Fig. 5(b)), it can be deduced that the sequence of precipitation events (crystallization and/or liquid-liquid phase 

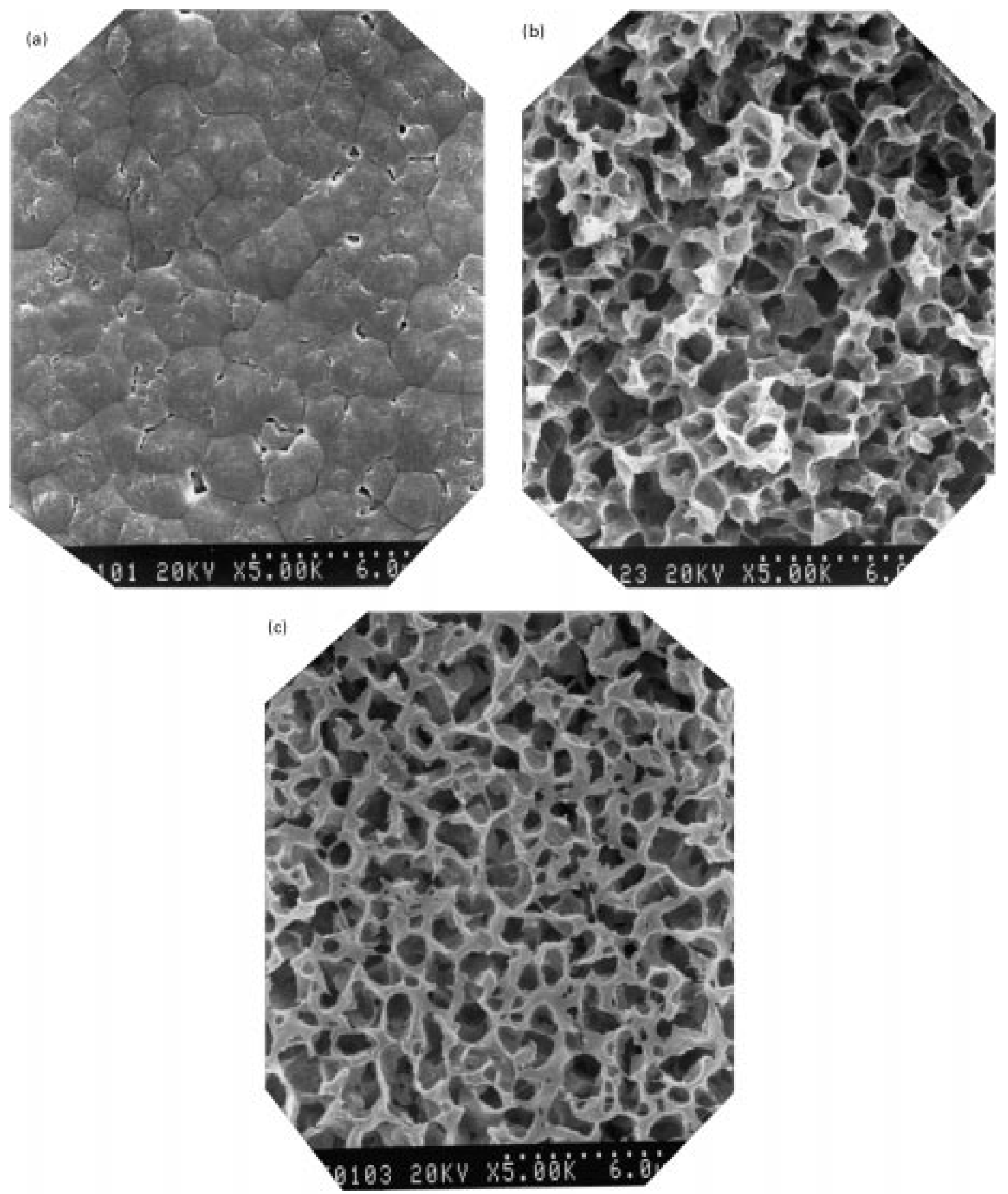

Fig. 5. SEM photomicrographs of the membrane prepared by immersing dope 'A' in bath II. (a) top view; (b) edge view; (d) bottom view.

separation) depends as much on the softness of the bath as on the aggregation state of the dope. Dope "A" was a good solution in which polymer chains were very well solvated by formic acid through a protonation mechanism [5]. By contrast, dope "B" was in a metastable state with respect to crystallization in which the polymer coil was on the edge of collapsing. In other words, nylon-610 was deprotonated and the polymer molecules began to associate into an aggregated structure. Such a dope contained a large population of subcritical crystal embryos (an aged dope turned turbid and upon heating it became clear again) $[1,2,9]$. As a result, crystallization could occur upon slight concentration 

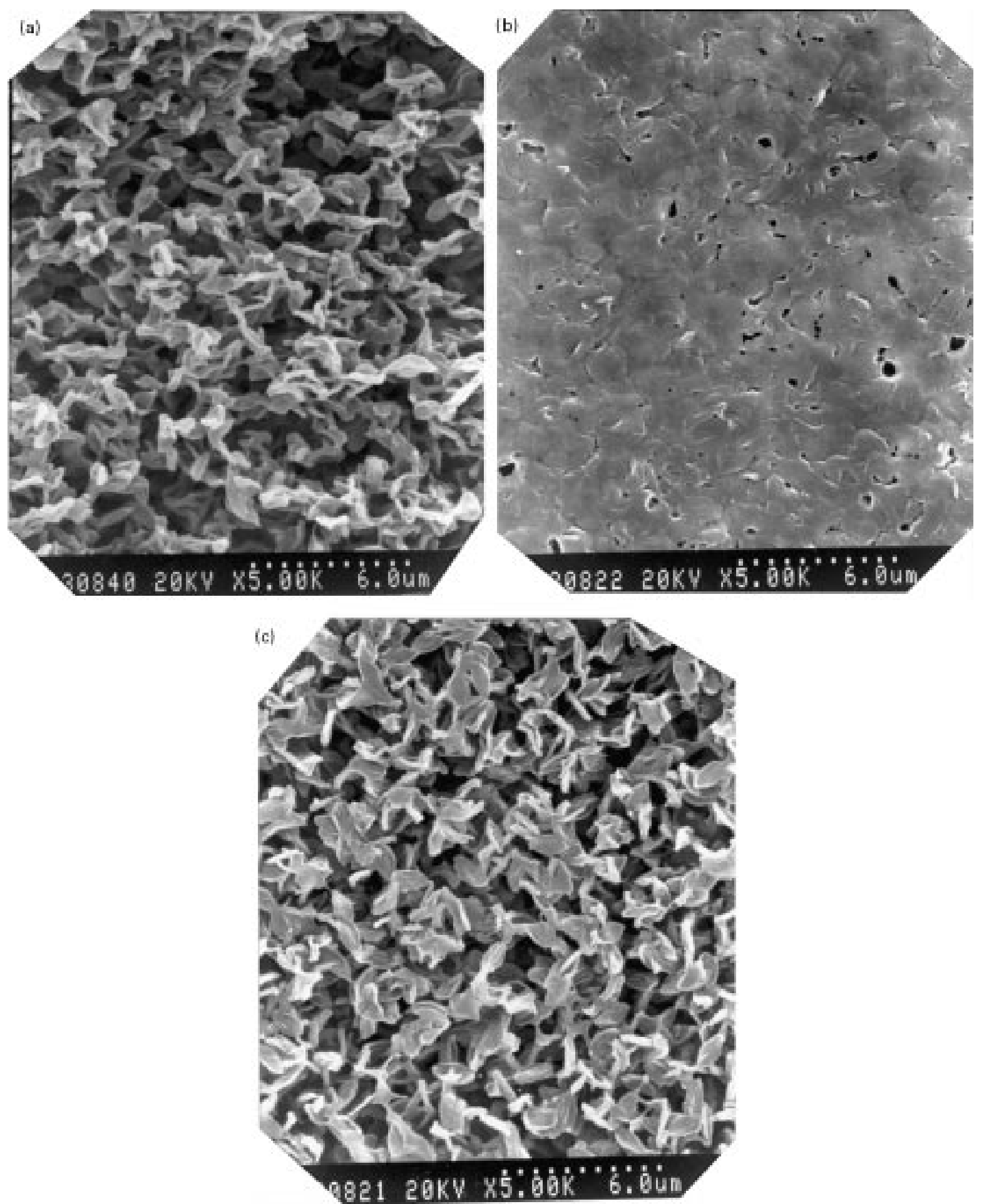

Fig. 6. SEM photomicrographs of the membrane prepared by immersing dope B in bath II. (a) edge view; (b) top view; (c) bottom view.

fluctuation caused by immersion of the dope into the bath; at which time, binodal miscibility boundary has not yet been entered.

The top surface of this membrane developed into a flattened structure with some small random crevices, as shown in Fig. 6(b). The crystallites appear to be smaller than those in Fig. 5(a) and some of them have the shape of a "sheaflike" spherulite representing an intermediate stage toward growth of a full spherulite [13]. This implies that there was a higher nucleation density in this top surface than that in Fig. 5 (a), although both membranes had similar polymer concentrations in the top surface region during the precipitation 


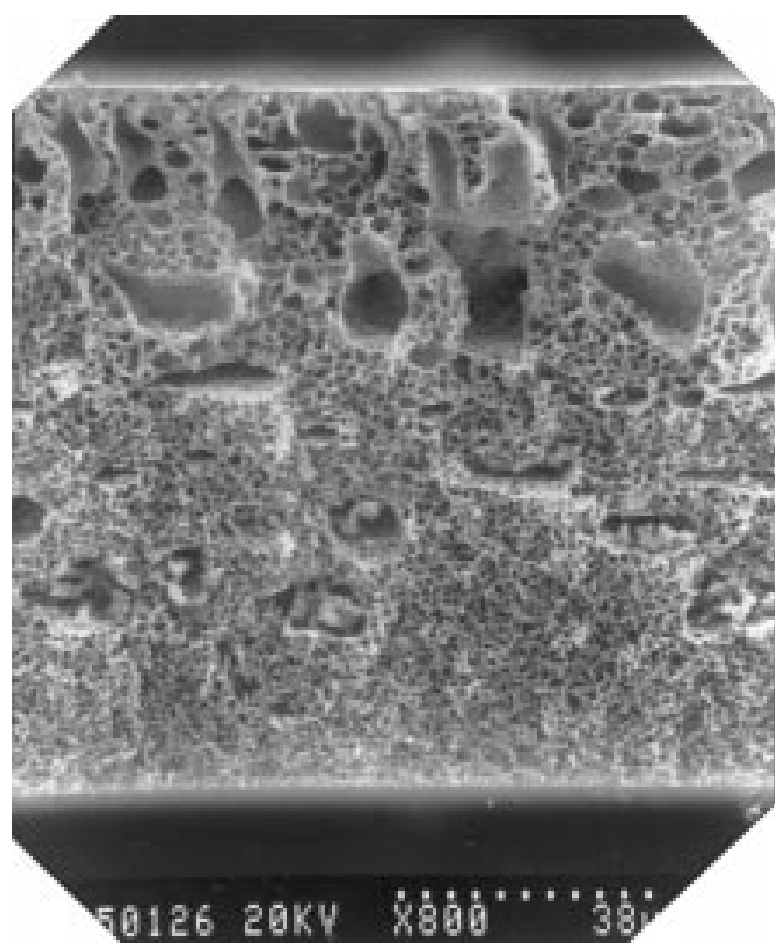

Fig. 7. The cross section of the membrane prepared by immersing dope $\mathrm{C}$ in bath $\mathrm{I}$.

process. Such a result is consistent with the dope's aggregation state for these membranes, as described above. The structure of the bottom surface, as shown in Fig. 6(c), is largely identical to the membrane bulk region. Unlike the flattened and partly closed bottom surfaces shown in Figs. 2(c) and 5(c), the stick-like crystallites here stand straightup and the surface is totally open. As a result of its higher micro-capillary force, this bottom surface is more wettable than those shown in Figs. 2(c) and 5(c). The wetting time of these membranes were measured to be $40 \mathrm{~s},>5 \mathrm{~min}$., and 1 min., respectively.

When a dope with a very high water content (e.g., point " $C$ " in Fig. 1) is immersed in bath I, the morphology of the membrane changes dramatically. It becomes highly anisotropic and large macrovoids are formed, as is observed from the SEM photomicrographs shown in Fig. 7. From light transmission experiments (see Table 1), it is clear that precipitation in this high water-content dope occured instanraneously after immersion. This result agrees with that reported by Smolders et al. who performed a similar study on the cellulose acetate membrane formation systems [15].

Table 1

Onset of precipitation in various dope/bath conditions

\begin{tabular}{lccc}
\hline Onset time (s) & Dope A & Dope B & Dope C \\
\hline Bath I & 9 & 5 & $<1$ \\
Bath II & 16 & 10 & 4 \\
1-Octanol & $>180 \mathrm{~s}$. & - & - \\
\hline
\end{tabular}

It is generally understood that macrovoids are formed in rapid precipitation conditions $[14,15,24]$. In the present case, the composition of the dope was close to the binodal (Fig. 1); hence the diffusion path entered the binodal miscibility gap at the instant of immersion. Nucleation of liquid micells commenced and grew rapidly into the macrovoids.

Fig. 8(a) and (b) show the structures of membrane formed when dope "C" is immersed in bath II. The macrovoids line up parallel and extend toward the central region of the membrane. Beneath the macrovoids, is a uniform porous structure resembling that shown in Fig. 6. This structure represents an interesting case where liquid-liquid phase separation takes place first but crystallization catches up and later dominates the precipitation process. Fig. 8(b) demonstrates the magnification of a macrovoid. It appears that the wall of the macrovoid is porous composed of stick or sheaf-like crystallites same as the other part of the membrane. If crystallization had taken place prior to liquid-liquid phase separation during membrane formation, Nylon-610 would form stick or leaf-like crystalline particles distributing uniformly in the entire membrane. Furthermore, as crystallization started, it would be impossible for the crystallites to cluster together and reorganize their distribution from within their domains to form macrovoids, i.e., crystalline structure would be fixed in their locations to suppress the tendency of further liquid-liquid phase separation. This suggests that formation of macrovoids induced by liquid-liquid phase separation occurred first and shortly after this process, the polymer-rich phase in the vinicity of the demixed macrovoids crystallized prior to the vitrification. It was also possible that liquid-liquid demixing and crystallization occurred nearly concomitantly. In either case, the formed morphology exhibited features from both liquid-liquid phase separation and crystallization. This can be explained by the fact that dope " $C$ " has a higher degree of supersaturation than dope " $\mathrm{B}$ "; meanwhile dope " $\mathrm{C}$ " is also nearer the binodal than dope B. Thus, the competetion between crystallization and liquid-liquid demixing is intense yielding a membrane with macrovoids originated from liquid-liquid phase separation and the wall of the latter are composed of crystalline particles.

\subsection{Skinless membrane formation by precipitation in 1-octanol bath}

As manifested in previous sections, all of the Nylon-610 membranes precipitated from water-formic acid solutions have a top surface layer that is significantly tighter than the other parts of the membrane. Such a dense layer, often termed skin in the literature, is typical of amorphous membranes that widely used in ultrafiltration and reverse osmosis processes. Commercial microfiltration membranes such as Nylon-6 [1] -66 [2] and PVDF [25,26], however, require that the membrane should have a uniform porous structure in all dimensions (skinless, of course), and the size of the pores fall roughly within the range of $0.1-5 \mu \mathrm{m}$. In 

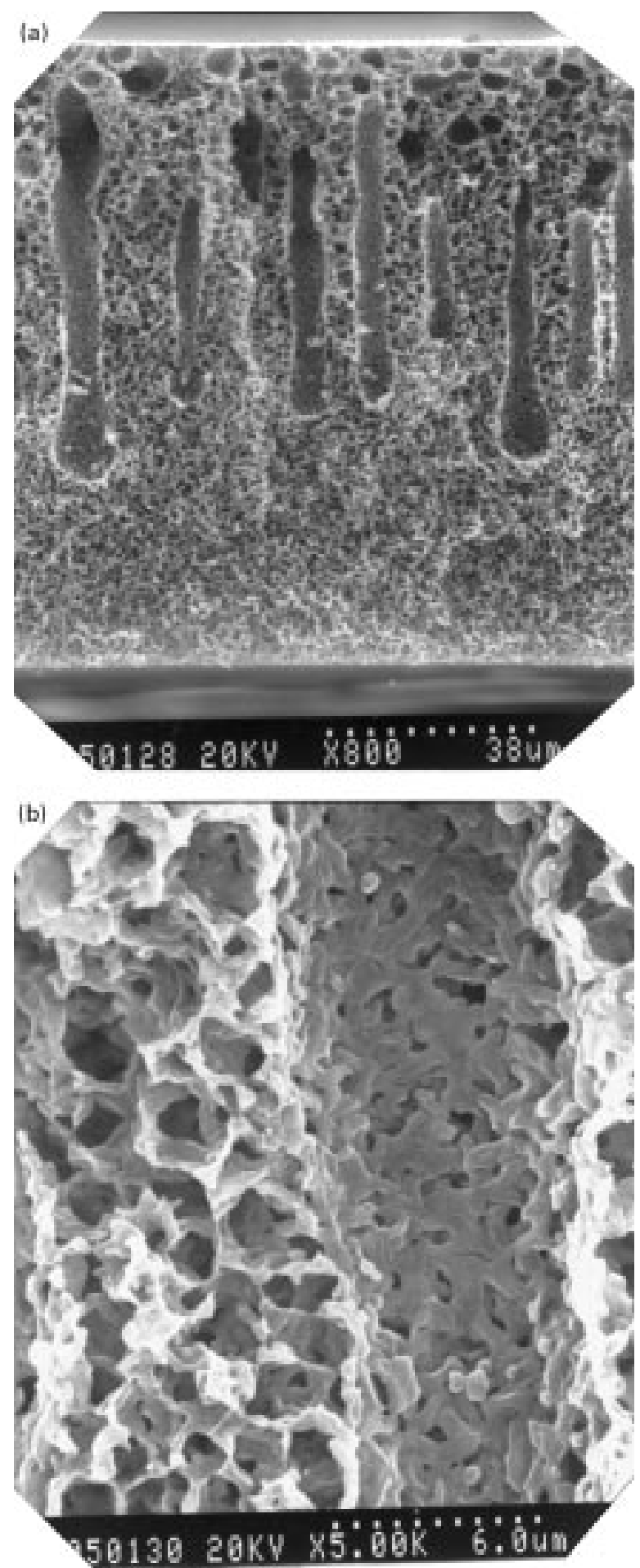

Fig. 8. SEM photomicrographs of the membrane prepared by immersing dope C in II. (a) edge view; (b) magnification of (a).

order to meet these demands, Nylon-610 membranes were prepared, using pure 1-octanol as the nonsolvent bath. Fig. 9(a) and (b) show, respectively, the top and bottom surfaces of the membrane made by immersing dope " $\mathrm{A}$ " in 1-octanol. Structure of the membrane's cross section resembles that of the top surface and is therefore not shown here. The top
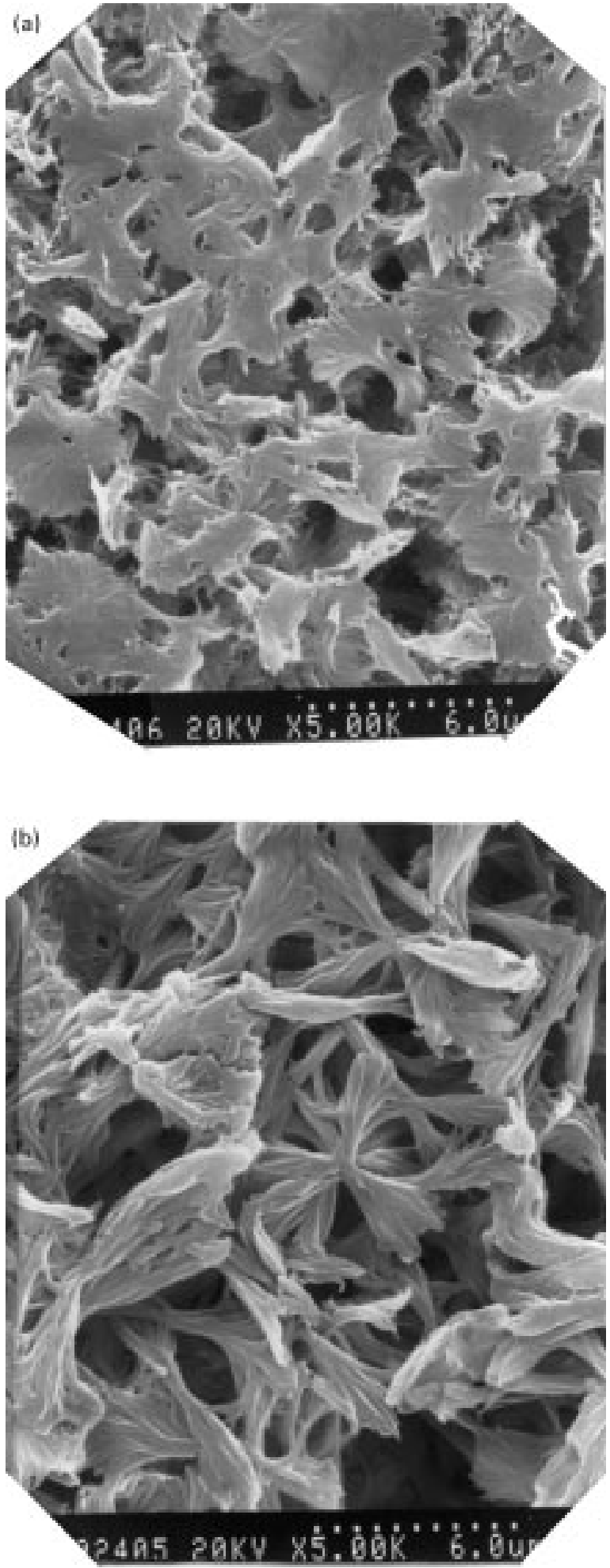

Fig. 9. SEM photomicrographs prepared by immersing dope A in 1-octanol. (a) top view; (b) bottom view.

surface is open composed of "sheaf-like" crystallites which interlock into a rather uniform porous structure whose pore diameter is on the average $3 \mu \mathrm{m}$. The sheaflike particles appears to be formed from parallel crystalline 
lamellae that splays out at the ends following a sperulitic growth habit [13]. The bottom surface is also composed of sheaf-like particles of the same size (same nucleation density as well) as the top surface and membrane bulk, although the particles here are somewhat flattened owing to their growth pressing against the glass surface. As liquid-liquid phase separation has been suppressed in this membrane, such a homogeneous porous structure suggests that the crystalline particles in the membrane were spawned in a similar concentration field and then dominated the precipitation process. Strathmann proposed a qualitative model to explain the causes of forming a uniform concentration profile in slow precipitation processes [14]. Also, in Smolders', McHugh's, and Cheng's models, flat concentration profiles have been predicted for slow precipitation cases. Therefore, the homogeneous porous structure shown here was made possible when phase separation process occurs sufficiently slowly (cf. Table 1) and the gap between the crystallization line and the binodal is large enough to prevent initiation of liquid-liquid phase separation.

\section{Conclusion}

In this work, formation of Nylon-610 membranes by isothermal immersion-precipitation from formic acid solutions in either water or 1-octanol nonsolvent baths was investigated. The prepared membrane were found to bear characteristics from crystallization and/or liquid-liquid phase separation, depending on the sequence of phase separation events that comprise the precipitation process. The following points are drawn from careful examination of the X-ray, DSC, SEM, and phase diagram results.

(1) When precipitation takes place in an aqueous bath, asymmetric membranes having a tight top surface are formed. At one extreme, when the dope is a good solution and the bath is pure water (i.e., harsh nonsolvent), liquidliquid phase separation precedes crystallization and a sponge-like structure composed of cellular pores is produced. At the other extreme, when the dope is in a prenucleation metastable state with respect to crystallization and the bath contains considerable amount of formic acid (i.e., soft nonsolvent), crystallization dominates the precipitation process to yield a bi-continuous structure intertwined by stick or sheaf-like spherulites. For the intermediate cases, e.g., immersion of an incipient dope in water, a mixed structure is obtained consisting of cellular pores whose walls are dendritic and particulate. (2) Finger-like macrovoids are formed in case that the dope contains a large amount of water (e.g., $12 \mathrm{wt} \%$ in the present study). Unlike usual macrovoids observed in amorphous membranes, the walls of the macrovoids here are porous and spherulitic resembling the other parts of the membrane. (3) When dope solutions are immersed in a 1-octanol bath, a skinless uniform porous membrane is obtained. The building elements of the latter are sheaf-like crystallites of roughly identical size.

\section{Acknowledgements}

The authors thank the National Science Council of Taiwan, R.O.C., for the financial support, Grant \# NSC 84-2216-E-032-004.

\section{References}

[1] Marinaccio PJ, Knight RA. US Patent 3,876,738, 1975.

[2] Pall DB. US Patent 4,340,479, 1982.

[3] Knight RA. US Patent 5,084,179, 1992.

[4] Kesting RE, Cunningham LK, Morrison MC, Ditter JF. Desalination 1983;46:343.

[5] Yao GW, Burford RP, Fane AG, Feil CJD, McDonogh RM. J Appl Polym Sci 1987;34:2399.

[6] Xu YF, Huang RYM. J Appl Polym Sci 1988;36:1121.

[7] Lai J-Y, Chu Y-H, Huang S-H, Yin Y-L. J Appl Polym Sci 1994;53:999.

[8] Cheng LP, Dwan AW, Gryte CC. J Polym Sci Polym Phys 1994;32:1183.

[9] Cheng LP, Dwan AW, Gryte CC. J Polym Sci Polym Phys 1995;33:211.

[10] Cheng LP, Dwan AW, Gryte CC. J Polym Sci Polym Phys 1995;33:223.

[11] Bulte AMW, Folkers B, Mulder MHV, Smolders CA. J Appl Polym Sci 1993;50:13.

[12] Young TH, Lai JY, You WM, Cheng LP. J Membr Sci 1997;128:55.

[13] Wunderlich B. Macromolecular physics, New York: Academic Press, 1973.

[14] Strathmann H, Kock K, Amar P. Desalination 1975;16:179.

[15] Smolder CA, Reuvers AJ, Boom RM, Wienk IM. J Membr Sci 1992;73:259.

[16] Radovanovic P, Stephen W, Hwang SK. J Membr Sci 1992;65:231.

[17] Lai JY, Liu MJ, Lee KR. J Membr Sci 1994;86:103.

[18] Smolders CA, Reuvers AJ, Boom RM, Wienk IM. J Membr Sci 1992;73:259.

[19] Kohan MI. Nylon plastics handbook, New York: Hanser Publishers, 1995.

[20] Brandrup J, Immergut EH. Polymer handbook, New York: Wiley, 1996.

[21] Reuvers AJ, Smolders CA. J Membr Sci 1987;34:67.

[22] Kesting RE. Synthetic polymeric membranes, New York: Wiley, 1985.

[23] Tsay CS, McHugh AJ. J Polym Sci Polym Phys Ed 1990;28:1327.

[24] Ray RJ, Krantz WB, Sani RL. J Membr Sci 1985;23:155.

[25] Grandine JD. US Patent 4,203,847, 1977.

[26] Grandine JD. US Patent 4,203,848, 1977. 